\title{
The PARAChute Project: Remote Monitoring of Posture and Gait for Fall Prevention
}

\author{
David J. Hewson, ${ }^{1}$ Jacques Duchêne, ${ }^{1}$ François Charpillet, ${ }^{2}$ Jamal Saboune,, ${ }^{2}$ Valérie Michel-Pellegrino, ${ }^{1}$ \\ Hassan Amoud, ${ }^{1}$ Michel Doussot, ${ }^{1}$ Jean Paysant, ${ }^{3}$ Anne Boyer, ${ }^{2}$ and Jean-Yves Hogrel ${ }^{4}$ \\ ${ }^{1}$ Institute Charles Delaunay, FRE CNRS 2848, University of Technology of Troyes, 10000 Troyes, France \\ ${ }^{2}$ UMR LORIA 7503, Université de Nancy, CNRS-INRIA, Campus Scientifique, BP 239, 54506 Vandoeuvre-lès-Nancy, France \\ ${ }^{3}$ Institut régional de Réadaptation, Faculté de medicine, 9 Avenue de la Forêt de Haye, BP 184, 54500 Vandoeuvre, France \\ ${ }^{4}$ Neuromuscular Physiology Laboratory, Institut of Myology, GH Pitié-Salpêtrière, 75651 Paris, France
}

Received 10 March 2006; Revised 19 October 2006; Accepted 21 February 2007

Recommended by Francesco G. B. De Natale

Falls in the elderly are a major public health problem due to both their frequency and their medical and social consequences. In France alone, more than two million people aged over 65 years old fall each year, leading to more than 9000 deaths, in particular in those over 75 years old (more than 8000 deaths). This paper describes the PARAChute project, which aims to develop a methodology that will enable the detection of an increased risk of falling in community-dwelling elderly. The methods used for a remote noninvasive assessment for static and dynamic balance assessments and gait analysis are described. The final result of the project has been the development of an algorithm for movement detection during gait and a balance signature extracted from a force plate. A multicentre longitudinal evaluation of balance has commenced in order to validate the methodologies and technologies developed in the project.

Copyright (c) 2007 David J. Hewson et al. This is an open access article distributed under the Creative Commons Attribution License, which permits unrestricted use, distribution, and reproduction in any medium, provided the original work is properly cited.

\section{INTRODUCTION}

The study of balance deficits is of interest for many reasons, in particular for people with various pathological conditions affecting balance and the elderly. In respect to an elderly population, falls are a major problem, in terms of both frequency and consequences. In France alone, more than two million falls are recorded among the elderly each year, leading to more than 9000 deaths [1]. Most prospective studies have attempted to identify risk factors, particularly in groups at high risk of falling [2-5]. The factors identified in these studies have often varied, mainly due to differences in methodology, diagnosis, and the study population [6]. Nevertheless, several factors are regularly cited, such as muscular weakness [6], a previous fall [5], or balance problems [2, 4, 7-10]. In addition, several factors that augment the risk of falling, such as visual, vestibular, or proprioceptive problems, can manifest themselves by adversely affecting balance [11-13]. In most of these studies, balance is measured using either clinical or biomechanical tests. Several different clinical tests exist, such as the Timed Get-up-and-go [14], the Berg Balance Scale [15], and the Tinetti Balance Scale [8], which can be used to predict the risk of falling. Even though these tests have demonstrated their capacity to identify the risk of falling in the following year, they are not able to identify progressive changes in fall risk. To this end, these tests are not suited to use as a daily test. A simple biomechanical test of balance [16] and several parameters derived from it, such as the area and the form of the displacement of the centre of pressure $[3,17]$, have also been able to predict falls. However, these measures have never been integrated into a home-based test.

With respect to gait analysis, the gait signature has essentially been used to identify individuals [18-20] or for classification and/or the determination of the type of gait $[21,22]$. More recently, gait has been used as a biometric trait for identification purposes [23]. The hypotheses related to gait analysis are generally very restrictive: fixed camera, gait at a constant velocity, a frontoparallel approach in relation to the camera, all of the subjects visible, constant luminosity, absence of distractions, and so forth. In contrast to the approach of the present study, these experiments have been performed in a laboratory setting and no real application has been demonstrated. 
Two major classes of methods are used for gait analysis: calculation of implicit periodic space-time models [18, 20, $23,24]$, which requires a history to be kept; and calculation of specific characteristics (velocity, cadence, stride length, etc.), usually in real time $[19,22]$. Even though these methods have not resulted in any practical applications, these studies have at least highlighted the concept of the signature on which the current methodology is based.

A risk evaluation, such as that outlined above, will be of no discernible benefit if it is not followed by a reduction in risk due to the intervention of a health professional. Many research teams have worked in this area over the last 10 years, and have reported the results of intervention studies on the risk of falling. Several multidisciplinary intervention studies have been found to be effective $[25,26]$, but the approach that has demonstrated the most potential is the adoption of an exercise program, either in a group $[27,28]$, or at home $[29,30]$. The benefits of an exercise program are related to the fact that the principal risk factors (muscular weakness and balance problems) are those which exercise programs have the greatest effect of [31]. In order for such programs to be put in place without costing too much, the programs need to be administered only to those people identified as having a high risk [30, 32]. Given such a requirement, the necessity for a simple and effective evaluation of balance is obvious.

The aim of the PARAChute project was to propose a methodology and a technology that would enable the detection of an evolution towards a risk of falling in communitydwelling elderly. The technique is based on evaluations of the quality of balance and gait.

The methodology used has to take into account the multiple constraints related to home-based testing.

(i) The evaluation system needed to be adapted to the home of the elderly person under supervision, without disturbing their typical environment.

(ii) The protocol needed to use typical daily activities.

(iii) The protocol should not require the presence of a third person.

(iv) All aspects of the system needed to preserve the privacy of the person. Irrespective of the data obtained, the information exiting the system should only be related to an evaluation of the risk of falling.

(v) The system needed to be able to function independently, as well as a part of a home-based vigilance network.

Balance was assessed using a miniature force plate, while gait was assessed using a video camera placed in a corridor of the home. The camera included an image analyzer, which ensured that rather than transmit images, only information on the gait signature was sent, thus preserving the privacy of the person being assessed.

The paper is organized as follows: Sections 2 and 3 describe the procedures for balance assessment and gait analysis, respectively. Each of these sections includes results and discussion related to the assessment method. In Section 4, the remote monitoring system is outlined, while in Section 5, conclusions and future work are presented.

\section{BALANCE ASSESSMENT}

Balance can be assessed using either clinical or biomechanical tests, as outlined previously. Given the requirement of a clinician to be present for clinical tests, only biomechanical tests were considered for remote assessment. Furthermore, those biomechanical tests that require the subject to undergo perturbation, such as dynamic posturography, are obviously unsuited to remote testing, due to the lack of supervision available [33]. The only biomechanical test of posture suitable for remote monitoring is static posturography, whereby subjects are required to step onto a force plate, remain stationary for a pre-defined period of time, before stepping down off the force plate. Such a test is similar to that required to weigh oneself using a bathroom scale, something that should be within the capabilities of an elderly person living in the community.

Although it is not possible to perform dynamic posturography due to the lack of supervision during the testing procedure, information related to dynamic posture can still be obtained from the force plate measurements. The initiation of a movement from a static posture needs postural equilibrium to be broken, thus requiring the generation of ground reaction forces (GRFs). These GRFs constitute a source of perturbation for postural equilibrium. In order to successfully perform a movement, the nervous system must control the destabilizing effect of force generation. It has been suggested that falls are most likely to occur in the elderly during stepping up or descending from a stair or a step. Almost all of the previous studies related to stair or step descent have mainly analyzed forward descent [3438]. However, it has been suggested that the effect of examining backward movement could enable the identification of otherwise undetected pathological locomotion that would have remained undetected by analysis of forward movement alone [39].

An analysis of the control of dynamic postural equilibrium in the elderly was therefore performed using the movement of stepping up and descending from the force plate used in the static study. The analysis of such a movement enabled the identification of parameters related to dynamic equilibrium, which could then be combined with more classical measures of static equilibrium in order to provide an overall evaluation of equilibrium.

\subsection{Static equilibrium}

Postural stability can be measured using a force plate, from which measures of centre of pressure (COP) displacement in anteroposterior (AP), mediolateral (ML), and resultant (RD) directions are obtained. The stabilogram is a representation of the centre of pressure displacement in AP and ML, and can also be expressed as a function of time (see Figure 1). The parameters that characterize static equilibrium are then extracted from the stabilogram signal.

The classical parameters that are typically extracted from such COP signals include temporal (mean, RMS), spatiotemporal (surface of the ellipse), and spectral parameters (median frequency, deciles), as detailed in [40]. More recently, 


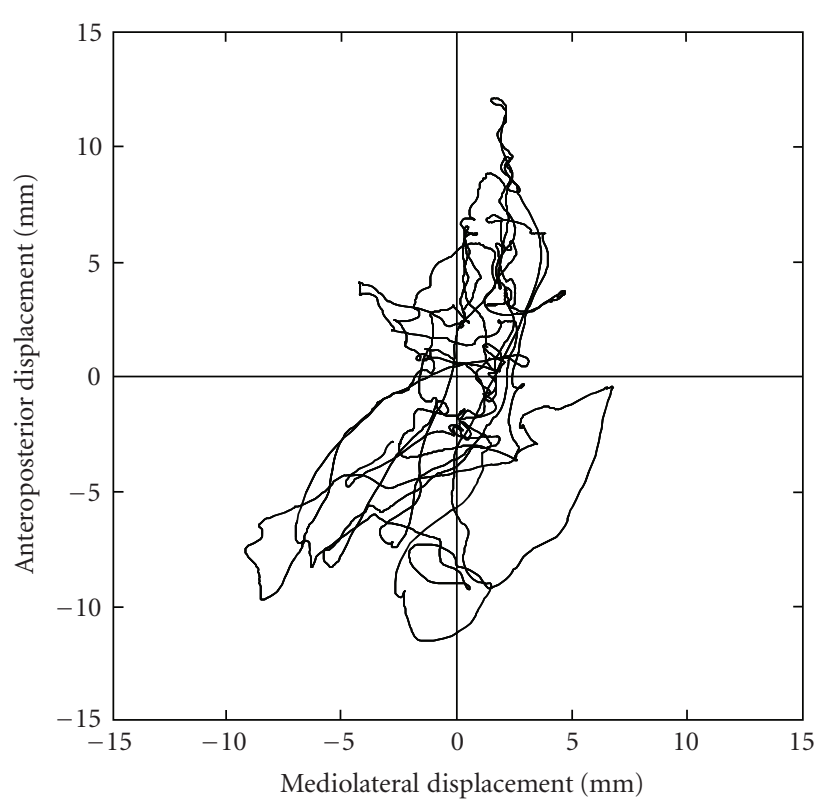

(a)

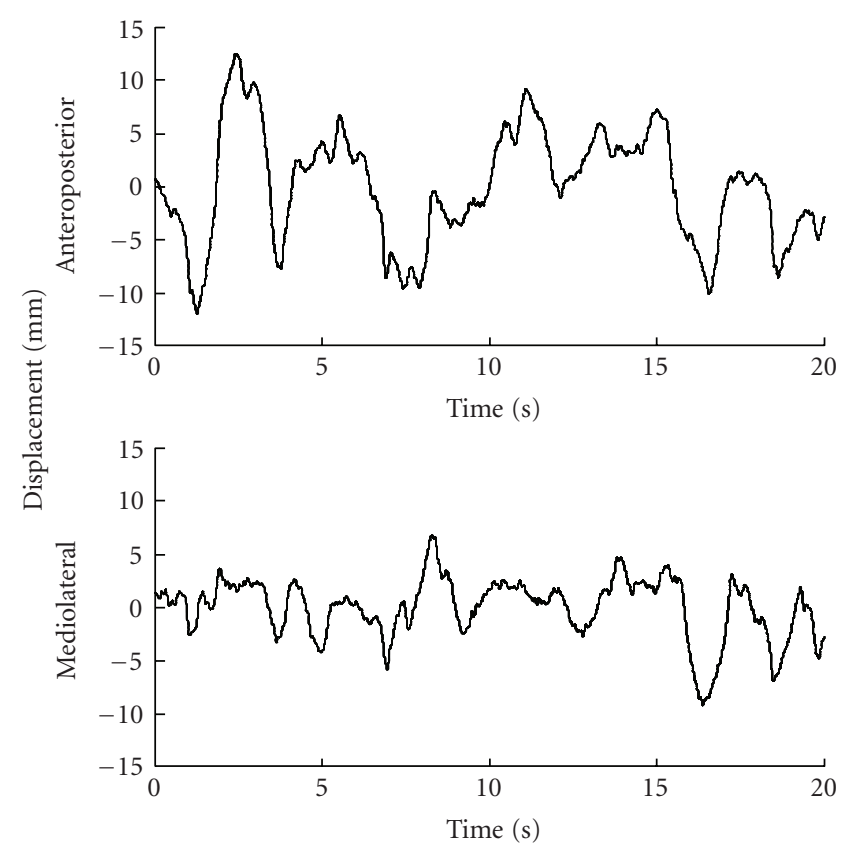

(b)

FIgURE 1: Typical stabilogram of the displacement of the centre of pressure (COP). Data are for a healthy 24 -year-old adult subject.

parameters linked to underlying physiological control systems have been identified to contain information related to long-term correlations and self-similarity. One of these parameters is the Hurst exponent $(\mathrm{H})$, which can be estimated using several methods: rescaled range analysis $(\mathrm{R} / \mathrm{S})$, detrended fluctuation analysis (DFA), and stabilogram diffusion analysis (SDA) [41, 42]. A high value of $\mathrm{H}$ indicates self-similarity, and a corresponding movement that is closely controlled. Such parameters might provide a means of following balance disorders longitudinally [43].

\subsubsection{Methods}

In order to compare the capacity of different estimates of the Hurst exponent to discriminate between elderly and adult subjects, an experimental study was performed. The Hurst exponent was estimated using SDA [41] and DFA [44]. In the present study, all time series were found to be fractional Brownian motion ( $\mathrm{fBm}$ ) after application of DFA. If the slope $\alpha$ obtained from DFA is greater than 1 , this indicates that the series is $\mathrm{fBm}$. It was not possible, therefore, to use the $\mathrm{R} / \mathrm{S}$ method, which can only be applied to fractional Gaussian motion [45].

Subjects were 90 healthy young adults ( 57 males, 33 females) and 10 healthy elderly ( 4 males, 6 females). For the young adults, the mean age, height, and weight were $19.7 \pm$ 0.8 years, $174.9 \pm 9.5 \mathrm{~cm}$, and $67.0 \pm 11.1 \mathrm{~kg}$, respectively. For the elderly subjects, the mean age, height, and weight were $80.5 \pm 4.7$ years, $165.6 \pm 7.0 \mathrm{~cm}$, and $71.9 \pm 9.9 \mathrm{~kg}$, respectively. All subjects who participated gave their written informed consent. No subjects reported any musculoskeletal or neurological conditions that precluded their participation in the study.

Subjects were instructed to look straight ahead, with their arms placed at their sides in a comfortable position, and were tested either barefoot or wearing socks. Upon a verbal command, subjects stepped onto a force plate (4060-80, Bertec Corporation, Colombus, Ohio, USA) with no constraint given over foot position. Subjects were instructed to look at a $10 \mathrm{~cm}$ cross-placed on a wall $2 \mathrm{~m}$ in front of the force plate. After 10 seconds, subjects stepped down backwards off the force plate.

Data were acquired with an NIDAQ card (6036E, National Instruments, Natick, USA) at $100 \mathrm{~Hz}$ with a lowpass Butterworth filter (8th-order, cutoff frequency $10 \mathrm{~Hz}$ ). The initial COP signals were calculated with respect to the centre of the force plate before normalization by subtraction of the mean. All calculations of COP data were performed with Matlab. The intraclass correlation coefficient (ICC) was used as a measure of reliability [46].

\subsubsection{Results}

The results for the SDA method are only for the short-term region of the $\Delta t$ by $\langle\Delta x\rangle^{2}$ curve used to calculate SDA. No significant differences were observed for the long-term region, with values often less than zero, making interpretation impossible. This could have been due to the short duration of the time series used in the present study (10 seconds) in keeping with the constraints of a home-based test.

A comparison of the SDA method for the two populations is presented in Figure 2. No significant differences were observed between groups for mediolateral (ML) displacement. However, elderly subjects had significantly greater values for both anteroposterior (AP) and the resultant (RD) displacement than the control subjects. 


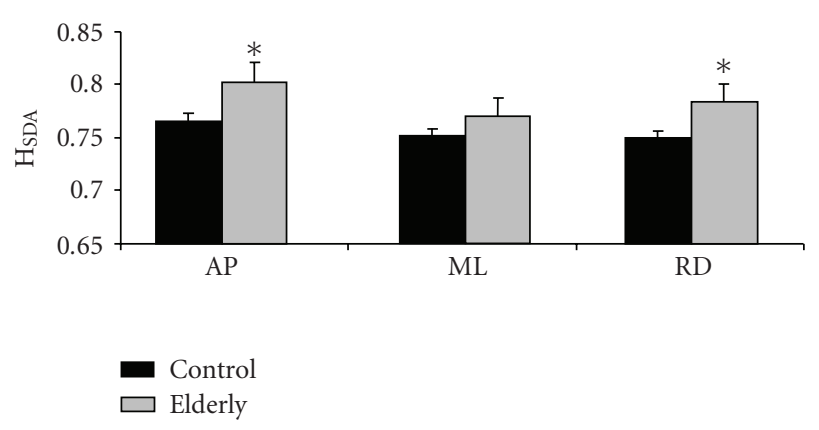

FIGURE 2: Estimation of the Hurst exponent using SDA for elderly and control subjects. Data are means and SD. $*$ denotes significant difference from control subjects.

A comparison of the DFA method for the two populations is presented in Figure 3. Significantly greater values were observed for elderly subjects for ML displacement. In contrast, elderly subjects had significantly smaller values for AP displacement.

\subsubsection{Interpretation of results}

As expected, the results of the SDA method showed higher values for elderly subjects, which are indicative of a less precisely controlled movement. The DFA method showed higher values for elderly subjects for ML displacement. In contrast, the DFA method yielded lower values for AP displacement for elderly subjects. DFA values less than 0.5 are indicative of antipersistence, with the lower the value, the greater the antipersistence, indicating a more closely controlled posture. Thus, elderly subjects were more stable than the control subjects for AP displacement. A possible interpretation for the greater stability observed for elderly subjects in the AP direction is that they controlled their movement in the AP direction more precisely, as identified by Norris et al. [47]. With respect to the results for AP displacement for SDA and DFA, the differences are due to the methods used. The short-term results for the SDA method are for short-term oscillations related to persistence, as all values were greater than 0.5 . The DFA results, for which values were less than 0.5 , are for the entire signal, which demonstrated antipersistence. Thus, the two methods provide information that can be considered complementary, with each method related to different aspects of postural control, for short-term and long-term autocorrelations for SDA and DFA, respectively.

\subsection{Dynamic equilibrium}

As mentioned previously, dynamic equilibrium is implicated in falls in the elderly. There are two approaches that could be used to calculate dynamic equilibrium during stepping up and climbing down, which are known as local and global biomechanical approaches. A local approach analyzes movement in terms of joint moments, muscle power modifications, and joint angular displacement $[38,48]$, whereas a

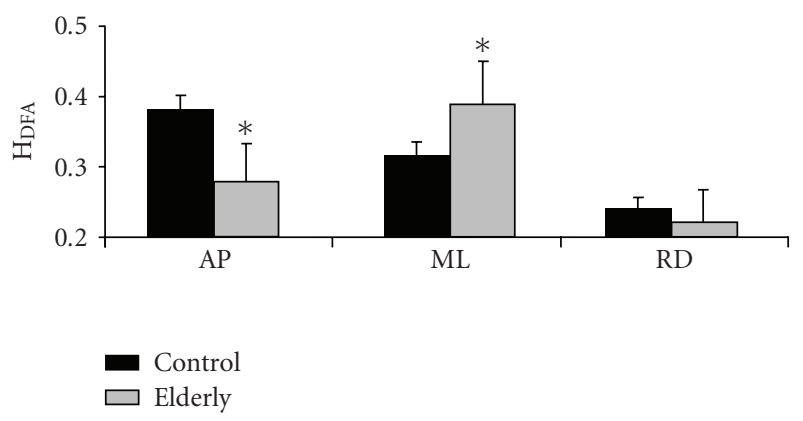

Figure 3: Estimation of the Hurst exponent using DFA for elderly and control subjects. Data are means and SD. $*$ denotes significant difference from control subjects.

global approach analyzes whole-body dynamics [49]. In the context of the present study, it would not be feasible to use a local approach due to the requirement for measures that cannot be obtained remotely. In contrast, a global approach requires only GRF, which can be obtained from the force plate in a remote setting. A detailed description of dynamic equilibrium obtained from force-plate measures can be found in $[50,51]$.

\subsubsection{Parameter selection}

The parameters chosen for this part of the study were those extracted from GRF (impulse, acceleration, and velocity of the CoM, slope of vertical GRF) relative to temporal parameters (durations of anticipatory postural adjustment, weight transfer, and swing phases) of the movement. Given that the protocol for static equilibrium required subjects to step onto a force plate, it seemed logical to measure dynamic parameters during the perturbation caused by this stepping-up movement. In addition, as it has been suggested that the effect of examining backward movement rather than forward movement enabled the identification of an otherwise undetected pathological gait, parameters were also extracted for the stepping-down movement from the force plate.

\section{Selected parameters for stepping up}

(i) Temporal parameters (see Figure 4). The total duration of the entire movement (dTOTAL) and the durations of the individual phases (WT: weight-transfer phase; SW: swing phase).

(ii) Ground reaction force parameters (see Figure 4). The impulsion of the reaction forces measured at the second footoff the ground (FO2) for all three axes of movement. The acceleration of the centre of gravity of the subject measured at FO2 for all three axes. The variation of the velocity of the centre of gravity of the subject measured at the second foot contact with the force plate (FC2) for all three axes. The loading rate of the lower limb (LR), taken as the mean slope of the ground reaction forces measured for the vertical axis and normalized by subject weight. 


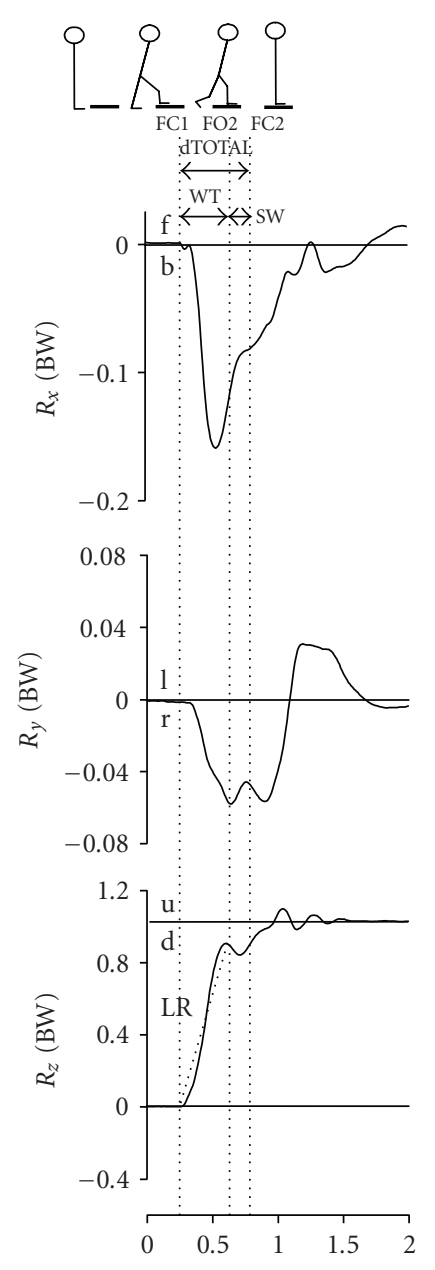

(s)

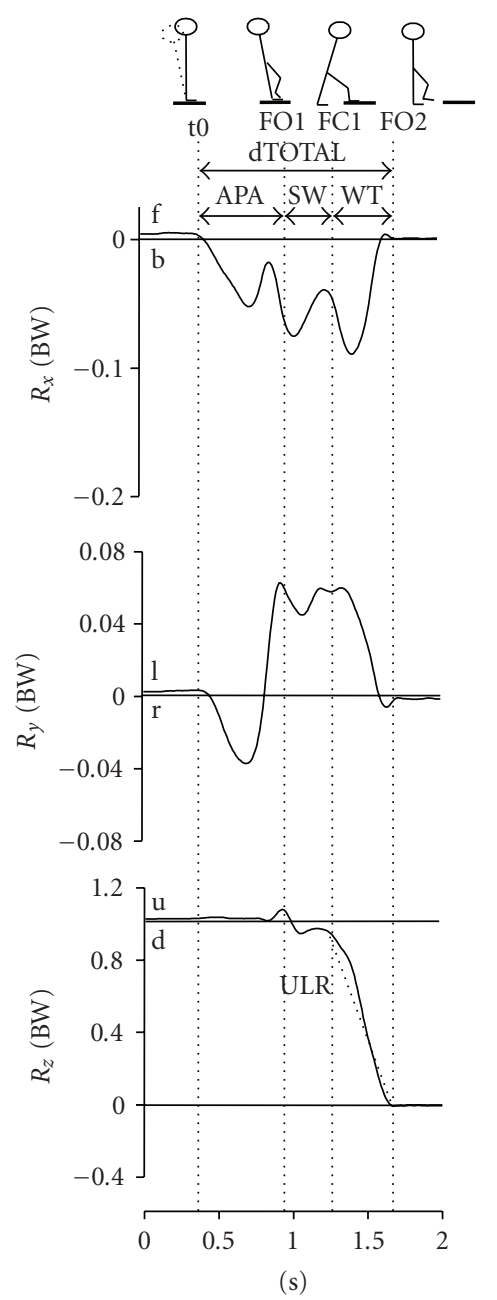

(b)

FIGURE 4: Biomechanical data obtained from a force plate for a typical control subject during stepping up and stepping down. $R_{x}, R_{y}, R_{z}$ : GRF normalized to body weight for AP, ML, and vertical directions. (f: forward, b: backward, l: left, r: right, u: upward, d: downward). (a) Stepping-up traces: FC1: first foot-contact on the step; FO2: second foot-off the ground; FC2: second foot-contact on the step; WT: weight-transfer phase; SW: swing phase; dTOTAL: stepping-up movement; LR: slope of vertical force. (b) Stepping-down traces: $t_{0}$ : first modifications of biomechanical traces; FO1: first foot-off from the force plate; FC1: first foot-contact on the ground; FO2: second foot-off from the force plate; dTOTAL: total duration of the backward stepping-down movement; dAPA: anticipatory postural adjustment duration; SW: swing phase; WT: weight-transfer phase; ULR: slope of vertical force.

(iii) Parameters related to the trajectory of the centre of pressure. The total length of the displacement of the COP for the resultant, as well as the individual movement directions (AP and ML).

\section{Selected parameters for the descent}

(i) Temporal parameters (see Figure 4). The total duration of the entire movement (dTOTAL) and the durations of the individual phases (APA: anticipatory postural adjustment; SW: swing phase; WT: weight-transfer phase).

(ii) Ground reaction force parameters (see Figure 4). The impulsion of the reaction forces measured at FO2 for all three axes of movement. The velocity of the centre of gravity of the subject measured at FO1 for all three axes. The GRF mea- sured at $\mathrm{FC1}$ for all three axes. The unloading rate of the lower limb (ULR), taken as the mean slope of the ground reaction forces measured for the vertical axis and normalized by subject weight.

(iii) Parameters related to the trajectory of the centre of pressure. The total length of the displacement of the COP for the resultant, as well as the individual movement directions (AP and ML).

\subsubsection{Methods}

Given that the dynamic parameters have not been used before, it was necessary to test them to ensure that they were able to distinguish between elderly and control subjects. To this end, two groups of subjects were analyzed: 11 control 


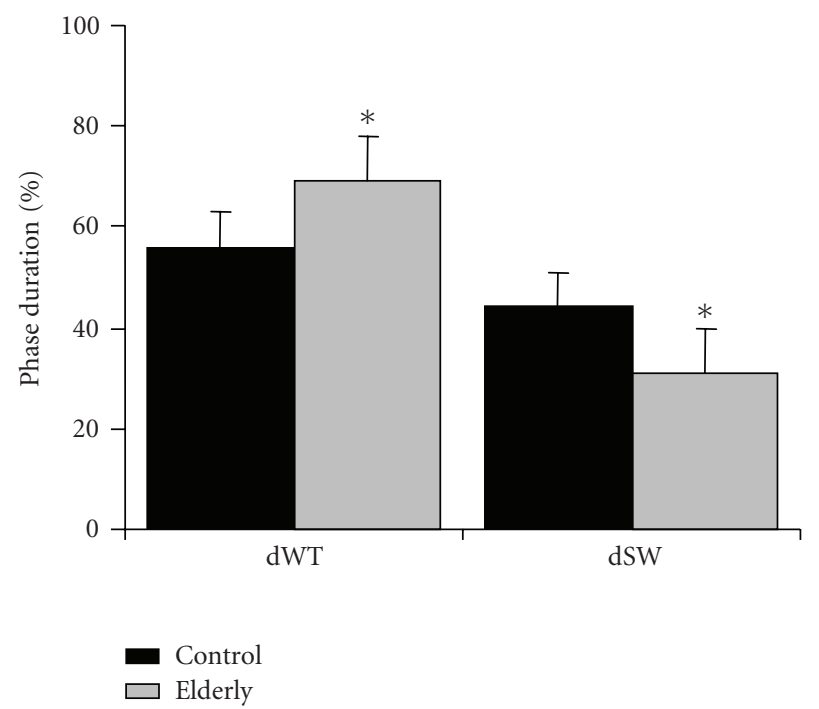

FIgURE 5: Temporal parameters during stepping up. dWT: relative weight-transfer phase duration, expressed as a percentage of total movement duration; dSW: relative swing-phase duration. $*$ denotes being significantly different from control subjects $(P<.05)$.

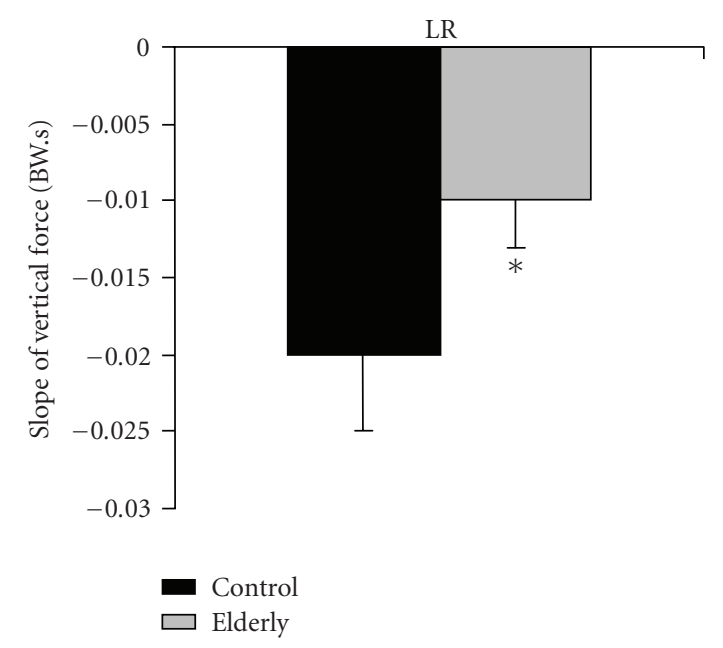

FIgURE 6: Slope of vertical force (LR) during stepping up. $*$ denotes being significantly different from control subjects $(P<.05)$.

(mean age $33.3 \pm 7.4$ years) and 14 elderly subjects (mean age $85.5 \pm 4.9$ years) were tested. The protocol used was the same as that described in Section 2.1.1. All temporal events were detected automatically using algorithms developed in Matlab (Mathworks Inc, Natick, Mass, USA). An analysis of the ability of the algorithms to automatically compute the time location of the various events was performed using an expert, who verified 40 trials in a pilot study, with a mean error of 0.03 seconds. Precise details of the methodology can be found in $[50,51]$.

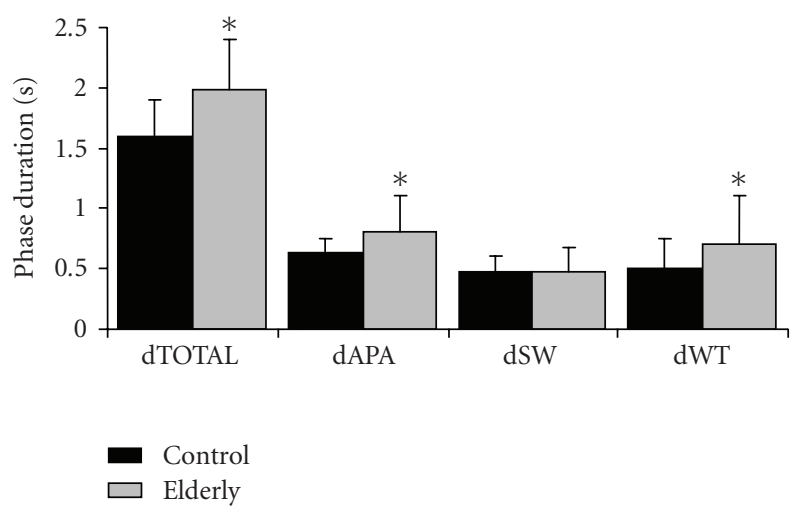

FIGURE 7: Temporal parameters during stepping down. dTOTAL: total movement; dAPA: anticipatory postural adjustment; dWT: weight-transfer phase duration; dSW: swing phase duration. $*$ denotes being significantly different from control subjects $(P<.05)$.

\subsubsection{Results}

The most important differences between elderly and control subjects for stepping up and stepping down are presented here.

\section{Stepping up}

With respect to the temporal parameters, elderly subjects spent more time in the weight-transfer phase and less time in the swing phase of the movement than did the control subjects (see Figure 5).

With respect to the GRF parameters, elderly subjects had a slower loading rate than control subjects (see Figure 6).

\section{Stepping down}

With respect to the temporal parameters, elderly subjects spent more time performing the movement due to the increased duration of the anticipatory postural adjustment and weight-transfer phases (see Figure 7).

In respect to the GRF parameters, elderly subjects had markedly lower anteroposterior CoM velocity than control subjects (see Figure 8). Unloading rate was also lower for elderly subjects (see Figure 9).

\subsubsection{Interpretation of results}

Elderly subjects were found to use different motor strategies in order to achieve the same movement as the control subjects both for stepping up and stepping down from the force plate. The principal differences were that elderly subjects decreased the duration of the swing phase, the moment when postural stability is the most precarious, while increasing the duration of the stance phase when posture is more stable. In addition, elderly subjects reduced the intensity of the perturbation forces, thus adopting a more precautionary approach to stepping up than control subjects. With respect to the descent, elderly subjects also adopted a more 


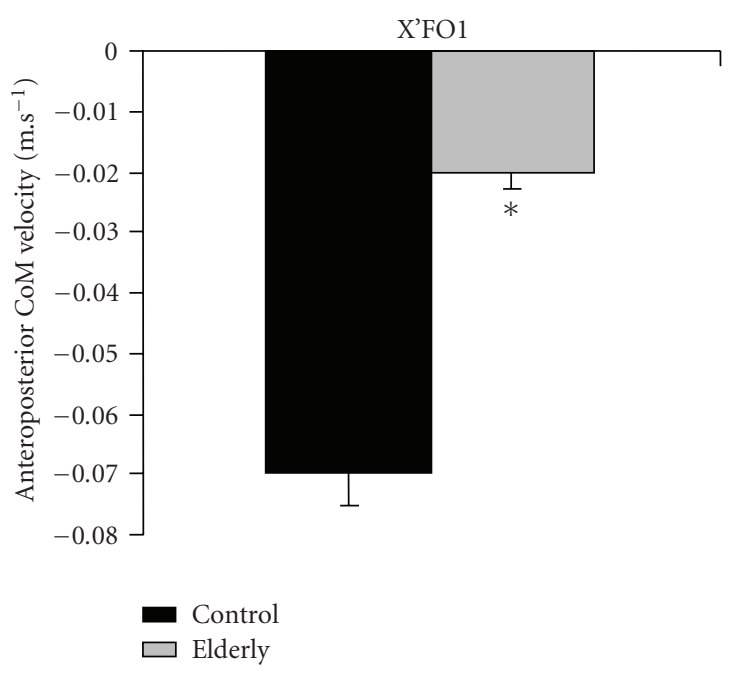

FIGURE 8: Anteroposterior CoM velocity at foot-off during stepping down. $*$ denotes being significantly different from control subjects $(P<.05)$.

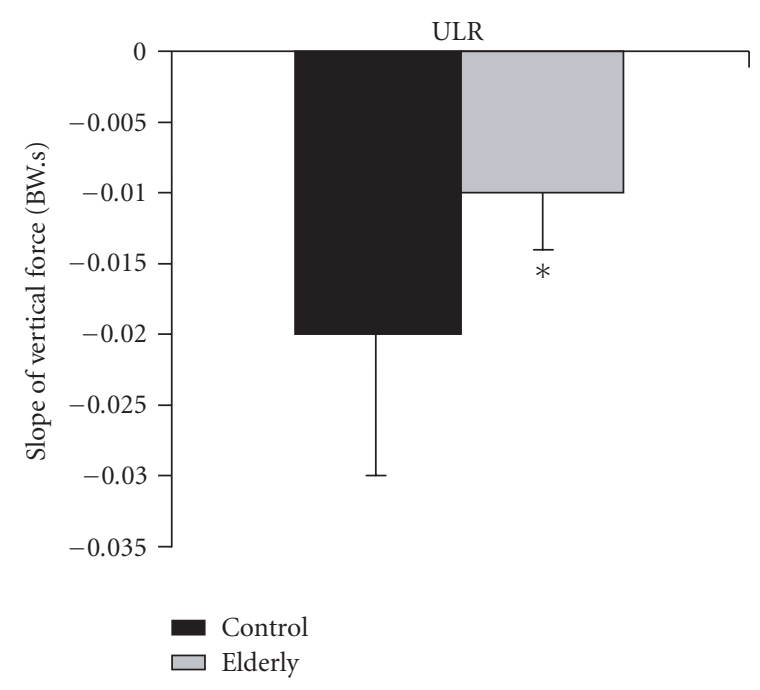

FIGURE 9: Slope of vertical force during stepping down. $*$ denotes being significantly different from control subjects $(P<.05)$.

precautionary approach than control subjects, as shown by the decreases in the acceleration of the centre of gravity, the GRF, and the unloading rate.

\subsection{Reliability}

There were a number of issues that needed to be addressed related to the testing protocol, in order for such a test to be feasible in a remote setting. Given that subjects will be used as their own reference, it is important that measures are reliable between tests. In a laboratory setting, it is possible to precisely control the measurement protocol, such that subjects' foot position and stance are almost identical between tests. Obviously, such a constraint is not possible in a remote setting, where subjects are free to choose their foot position and stance. Furthermore, precise information on the stance adopted by the subject is not available. To this end, a reliability study was performed. The subjects were those described in Section 2.1.1. Subjects were tested four times in order to determine reliability between testing sessions. The intraclass correlation coefficient (ICC) was used as a measure of reliability [46].

\subsubsection{Results}

The ICC values for the static variables ranged from 0.40 to 0.91 , with $70 \%$ of the values exceeding the 0.7 value considered to represent a "good" correlation [52]. With respect to the ICC values for the dynamic variables, values ranged from 0.66 to 0.95 , with $91 \%$ of the values exceeding 0.7 . The reliability values for ML displacement were generally greater than those for AP, which is not surprising, given that subjects' displacement varies more in an AP direction than in an ML direction due to the constraints on the system imposed by the ankle and knee joints. With respect to the reliability observed in previous studies, the present values are broadly in agreement. Lafond et al. reported ICC values for temporal, spatiotemporal, and spectral parameters that ranged from 0.22 to 0.87 for 30 -second recordings [53]. However, only those ICC for COP velocity exceeded 0.5 . In keeping with these results, the ICC values reported by Du Pasquier et al. for COP velocity was 0.79 for both displacement directions [54]. In one study in which the reliability of SDA parameters was assessed, Chiari et al. reported ICC values ranging from 0.41 to 0.79 , in keeping with the values reported in the present study [55].

There were major methodological differences between the studies cited above, and the present study in relation to foot position, recording duration, the time between tests, and the total number of tests. In all of the studies cited above, each subject's foot position was noted for the first trial, and all subsequent tests were performed using an identical foot position. In contrast, subjects in the present study were left to choose their foot position. It would have been expected that this freedom over foot position would have adversely affected the ICC reported. However, the ICC values reported were of a similar magnitude, irrespective of foot position. With respect to the duration of measurement, the present study used 10 -second times series, far shorter than that used previously, whereby ICC values were calculated for 30 seconds $[53,54]$, 50 seconds [55], or even 120 seconds [56].

An additional difference concerned the time taken between measures, which was 14 days between the first and last tests in the present study. In contrast, only the study of Corriveau et al. left a similar (up to 7 days) time period between tests. Other studies used rest periods up to three minutes between tests [53-55]. Finally, the number of tests used to obtain the ICC estimation varied from two $[54,56]$ to 9$10[53,55]$, in contrast to the four tests used in the present study. It is a well-known property of ICC values that an increased number of tests will produce an increase in the value observed. 


\subsection{Parameter selection and detection of a degradation in equilibrium}

The method best suited to detect a degradation in balance quality appears to be support vector data description (SVDD), which was developed by [57-61]. This method is based on the support vector machines of Vapnik [62], which finds the optimal separating hyperplane between data sets. In contrast, SVDD finds the sphere of minimal volume (or minimal radius) containing all (or most of) the objects. For a data set containing $N$ data objects, $\{x 1, \ldots, x N\}$, it is necessary to solve the following equation in order to find the sphere described by the centre $a$ and the radius $R$ that contains the most objects:

$$
\begin{gathered}
\min _{R, \xi_{i}} R^{2}+C \sum_{i=1}^{N} \xi_{i}, \\
\text { with }\left(x_{i}-a\right)^{T}\left(x_{i}-a\right) \leq R^{2}+\xi_{i} \quad \forall i, \xi_{i} \geq 0,
\end{gathered}
$$

where $\xi_{i}$ are slack variables, the variable $C$ gives the tradeoff between simplicity (or the volume of the sphere) and the number of errors (the number of target objects rejected). The dual Lagrangian problem of (1) will be

$$
\begin{gathered}
\min _{\alpha} \sum_{i, j} \alpha_{i} \alpha_{j}\left\langle x_{i}, x_{j}\right\rangle-\sum_{i} \alpha_{i}\left\langle x_{i}, x_{i}\right\rangle, \\
\text { with } \sum_{i} \alpha_{i}=1, \quad 0 \leq \alpha_{i} \leq C \forall i .
\end{gathered}
$$

Equality in (1) is satisfied for only a small set of objects, which are those on the boundary of the sphere itself. These objects, for which the coefficients $\alpha_{i}$ will be nonzero, are called support objects, and are all that is needed to describe the sphere. The radius $R$ of the sphere can be obtained by calculating the distance from the centre of the sphere to a support vector with a weight smaller than $C$. Those objects for which $\alpha_{i}=C$ are outside the sphere, and are considered to be outliers. To determine whether a test point $z$ is within the sphere, the distance to the centre of the sphere has to be calculated. A test object $z$ is accepted when this distance is smaller than the radius, that is, when $(z-a)^{T}(z-a) \leq R^{2}$. Expressing the centre of the sphere in terms of the support vectors, the objects $z$ is accepted when $f(z)$ is positive:

$$
f(z)=R^{2}-\sum_{i, j} \alpha_{i} \alpha_{j}\left\langle x_{i}, x_{j}\right\rangle+2 \sum_{i} \alpha_{i}\left\langle z, x_{i}\right\rangle-\langle z, z\rangle .
$$

To generalize the method to be used with kernels, the inner products of objects $\left\langle x_{i} \cdot x_{j}\right\rangle$ can be replaced by a kernel function $K\left(x_{i}, x_{j}\right)$, which implicitly maps the objects $x_{i}$ into some feature space. When a suitable feature space is chosen, a tighter description can be obtained.

Therefore, all inner products $\left\langle x_{i} \cdot x_{j}\right\rangle$ are replaced by $K\left(x_{i}, x_{j}\right)$ and the problem of finding a data domain description is now given by

$$
\begin{gathered}
\min _{\alpha} \sum_{i, j} \alpha_{i} \alpha_{j} K\left(x_{i}, x_{j}\right)-\sum_{i} \alpha_{i} K\left(x_{i}, x_{i}\right), \\
\text { with } \sum_{i} \alpha_{i}=1, \quad 0 \leq \alpha_{i} \leq C \forall i .
\end{gathered}
$$

A test object $z$ is accepted when $f(z)$ is positive:

$$
f(z)=R^{2}-\sum_{i, j} \alpha_{i} \alpha_{j} K\left(x_{i}, x_{j}\right)+2 \sum_{i} \alpha_{i} K\left(z, x_{i}\right)-K(z, z) .
$$

Given that each subject will act as its own reference, one-class SVDD will be used. More than 100 variables have been identified to characterize the equilibrium of a person, with those related to the organization of the COP trajectory of particular interest for a method based on self-learning. Such a large number of variables will need to be reduced using feature selection, before application of the SVDD model. The choice of a one-class model will require the use of nonsupervised feature selection to decrease the number of parameters in the model. In this way, it should be possible to identify subjects whose signature has changed in comparison to the model learnt during the initial phase of self-learning.

\subsubsection{Results}

The robustness and the sensitivity/specificity of the system are currently being evaluated as part of a two-year clinical trial. This trial will determine those parameters that are sensitive to changes in the equilibrium of the subjects studied. The system has already been tested with data for subjects who had an invoked degradation in postural equilibrium by means of vibration applied to the tibialis anterior tendon [63]. Vibration was applied bilaterally to the tibialis anterior tendon for 10 seconds using the VB115 vibrator (Techno Concept, Cereste, France). Subjects were then tested postvibration. Preliminary results using SVDD have shown the system to be $100 \%$ accurate at detecting a degradation in equilibrium. It should be noted that the magnitude of any degradation in elderly subjects is likely to differ from that in the artificially-invoked procedure detailed above. Nevertheless, the initially results are promising with respect to the future application.

\section{GAIT ANALYSIS}

The aim was to analyze gait quality in order to detect an evolution towards a risk of falling. The gait analysis system conceived for the project was developed to analyze the gait of an elderly person by the means of one or more cameras installed in their everyday living environment. Most gait analysis systems such as Vicon (Vicon Peak, Lake Forest, Calif, USA), use markers placed on the subjects at specific points, such as the knee and ankle joints. These markers are then detected by infrared cameras positioned in precise locations in the environment in which the person moves. A triangulation system is then used to reconstitute the position of these markers in space, from which it is possible to follow the trajectory of these points. Clinical conclusions can then be drawn on the quality of the gait of the person studied. These high-tech systems are expensive and have multiple constraints over their use. For instance, markers need to be placed on the subject, clothing must be reduced to a minimum, and an operator is required at all times. Given these constraints, such a system 


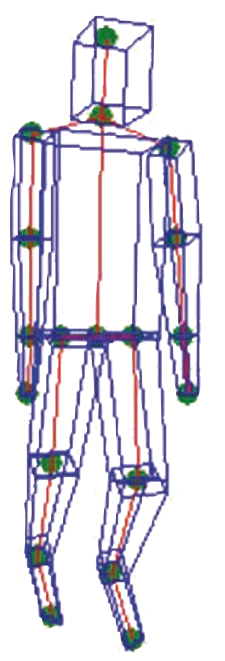

Figure 10: The articulated body model defined by 19 points and 17 segments.

is not suitable for a home-based test. However, the functionalities of such systems are of interest, as the richness of the information obtained enables most of the gait analysis parameters cited in the literature to be measured. To this end, it was decided to develop a similar system, without the need to position markers on the body, using low-cost video cameras.

\subsection{Methodology}

A detailed description of the methodology can be found in [64]. Subjects were taken as their own control, which required the identification of any significant variation in gait parameters that could predict fall risk. As in most 3D markerless motion capture systems, a 3D articulated body model was used (see Figure 10). This model is formed by 19 points representing key points of the human body (head, elbows, knees, etc.). These points are joined up by 17 segments modelling the human body. In order to simulate the way a human body moves, each of these segments was given a number of degrees of freedom (DOF) based on the rotation about $3 \mathrm{D}$ axes. The total number of DOF for the model used was 31. The proportion of the dimensions of the different body parts to the body's height was established using the "Vitruvian man" model of Leonardo da Vinci. Thus, the articulated model's dimensions were adapted to the height of the person tracked. The 3D positions of the 19 points in the model were calculated knowing the $31 \mathrm{DOF}$ and the $3 \mathrm{D}$ position of a particular point, termed the body origin. This approach can be qualified as simple and generic since neither dynamic modelling nor trained body-models were used.

The articulated model's configuration (established through its degrees of freedom) is then evaluated in order to determine the closeness of fit to the real body pose in the image using a likelihood function. A silhouette image of the tracked person is constructed by subtracting the background from the current image (video feed) and then by applying a threshold filter. This image is then compared to a synthetic

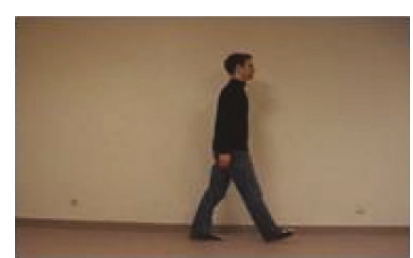

(a)

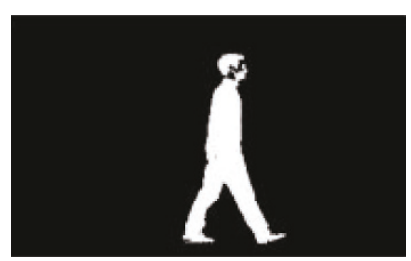

(b)

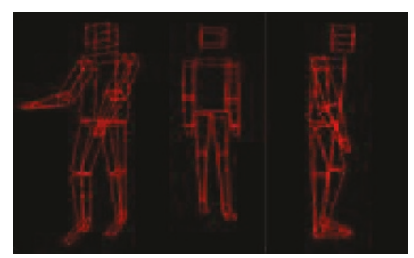

(c)

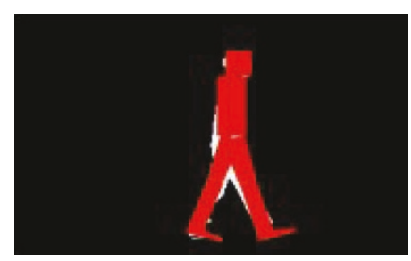

(d)

FIGURE 11: Estimation of the likelihood of the model. (a) The real image is obtained using a digital video camera. (b) The silhouette is extracted. (c) The virtual model is calculated. (d) The model is compared with the silhouette.

image representing the $2 \mathrm{D}$ projection of the $3 \mathrm{D}$ model configuration to which the likelihood is to be assigned (see Figure 11). This chosen method is simple, although it can be less effective when a person has loose clothing and in the presence of heavy shadows or poor lighting. These latter problems can partly be solved by adjusting the threshold filter or by applying a shadow suppression filter based on HSV color information [65].

The estimation of the $3 \mathrm{D}$ positions of the 19 body points (motion tracking) is then performed by finding the model configuration that best fits (having the highest likelihood function value) the real body pose, as represented in the video feed (silhouette). This problem can be considered as a Bayesian state estimation. In fact, the configuration of the $3 \mathrm{D}$ model represents the state vector of the model, $X$. In addition to the state model, an observation $Z$ is defined, through which the likelihood of a state vector at $t=t_{k}$ is evaluated by calculating $P\left(Z_{k} / X\right)$. In this approach, the observation is the 
TABle 1: Comparison between the IPF and Vicon systems. Data are mean values for two subjects.

\begin{tabular}{l|ccc}
\hline Parameter & Vicon & IPF & Difference \\
\hline Mean walking speed $(\mathrm{m} / \mathrm{s})$ & 1.196 & 1.174 & $1.8 \%$ \\
Mean left-leg stride length $(\mathrm{m})$ & 0.509 & 0.500 & $1.8 \%$ \\
Mean right-leg stride length $(\mathrm{m})$ & 0.436 & 0.427 & $2.1 \%$ \\
\hline
\end{tabular}

image of the person being tracked, while the weight (likelihood) of a model's configuration $m$ represents the observation probability $\left(w^{(k)}(m)=P\left(Z_{k} / X=m\right)\right)$. A particle filtering or condensation algorithm was chosen as the state estimator due to its capacity to handle non-Gaussian and multimodal probability densities (as in the case of motion tracking). Particle filtering searches for the best-fitting particle (state model configuration) in a well-defined particle set created at each time step. The basic particle filtering algorithm needs a large number of particles to provide a good estimation, particularly in high-dimensional spaces, where an increased complexity could make such an algorithm inapplicable.

The interval particle filtering (IPF) used has some simple modifications to the condensation algorithm in order to adapt the particle-search space configuration, thus making it more efficient whilst preserving the advantages of a particle filter. The IPF uses the same three-step structure of the condensation algorithm. In the selection step, a reduced number of particles are chosen among the heaviest particles produced in the previous time step. During prediction, each of these particles is replaced by a number of particles covering a multidimensional interval of neighboring particles. This interval is formed in a deterministic way, in accordance with the evolution of each component of the state model. The measure step remains unaltered in IPF. Details about this algorithm can be found in [64].

The IPF algorithm (coded in C++ Builder) was applied with 4096 particles, in order to track the movement of normal subjects moving in an ordinary environment. Video feeds of around 6 seconds were captured at 25 frames/second using a single commercial digital camera (Sony DV). Processing was performed offline using a Pentium $43 \mathrm{GHz}$ PC. The image resolution used was $360 \times 288$ pixels and $20 \mathrm{sec}-$ onds of processing time were required per frame to find the body-part configuration using IPF. Although this processing speed is far from real time, the system developed is suitable for the requirements of the current study. No calibration is needed, although the initial distance of the tracked person to the camera is specified. The initial results obtained from the IPF system were then compared to results obtained simultaneously using a Vicon system [64].

\subsection{Results}

The number of strides taken by subjects during the sixsecond data collection period varied from six to eight, depending on gait velocity.

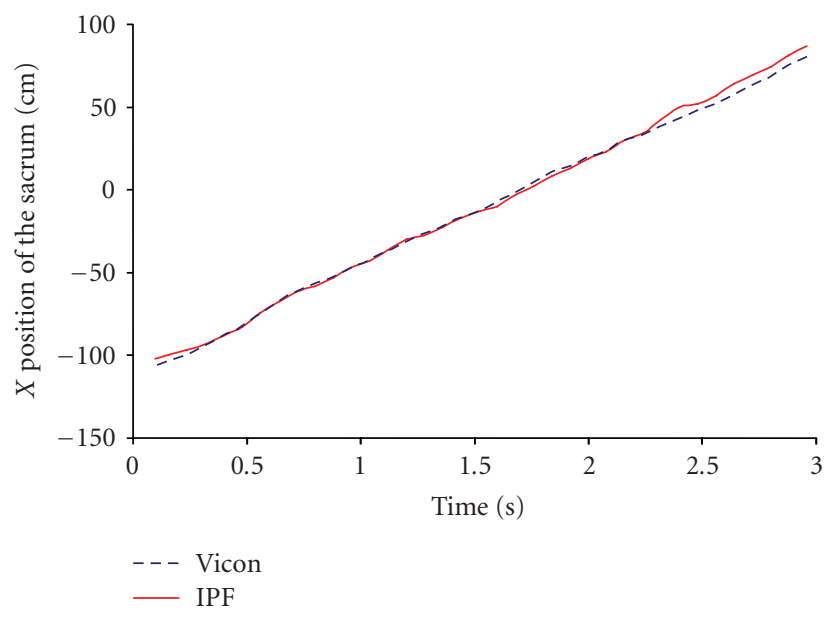

Figure 12: Comparison of $X$ position of the sacrum between the IPF and Vicon systems for a typical subject.

The comparison results between the IPF and Vicon systems can be performed using either the actual coordinates for the points of the 17-segment model, or by comparison of the extracted parameters. Typical results for the $x$ coordinate of the position of the sacrum, as identified by the two systems, are shown above (see Figure 12).

A comparison of the two systems for several parameters is shown in Table 1.

\subsection{Discussion}

Knowing 3D positions of the body's key points would enable the extraction of all classical gait parameters such as velocities, accelerations, stride length, stride width, and time of support. On the other hand, using a single camera would not provide accurate tracking of all points as some body parts would be occluded for a long portion of the video, depending on the view angle. The use of multiple cameras would solve this problem. Work is currently underway on developing a multicamera system. Another aspect currently being investigated is related to the extraction of new parameters, particularly those related to stride-time variability. Fractal analysis of fluctuations in gait rhythms has been shown to be related to fall risk and fear of falling in the elderly $[66,67]$. Such analyses typically need long time series, something which can prove difficult for elderly subjects. However, in a home-based test, repeated passages in front of the recording system might be able to be combined in order to provide longer time series. Work is currently underway in order to address this issue.

\section{REMOTE MONITORING SYSTEM}

The system consists of a local installation of a sensor and a local processing unit (LPU), which can communicate remotely with other parts of the surveillance network (see Figure 13). Precise details can be found in [68].

Although the final system needs to be lowcost, the initial design used the force plate technology outlined previously 


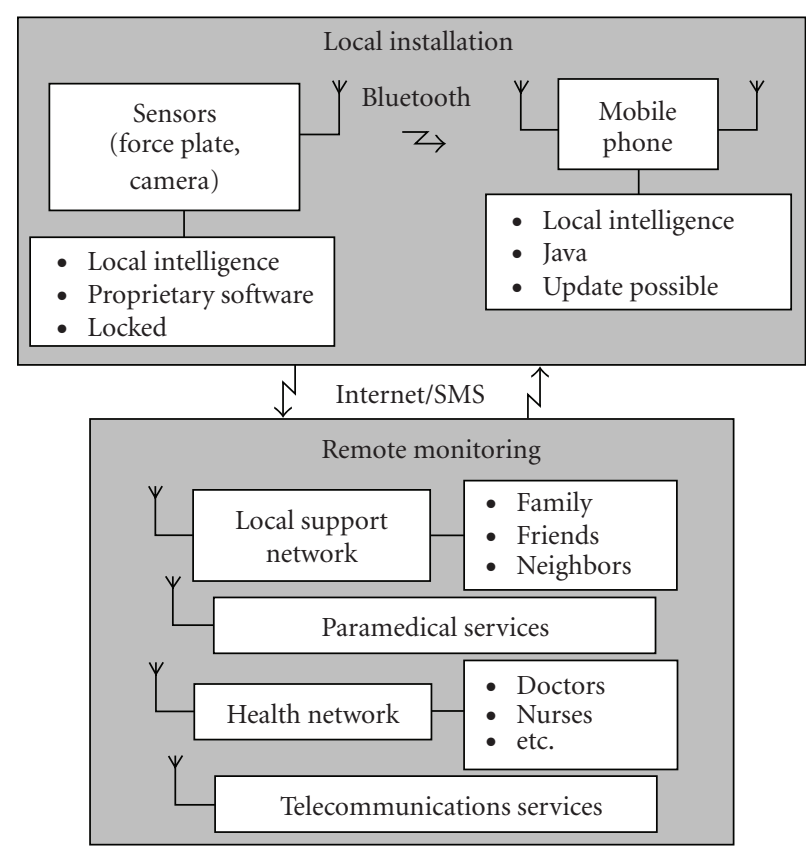

FIGURE 13: Schematic diagram of a remote monitoring system for balance assessment.

(4060-80, Bertec Corp., USA) fitted with a wireless Bluetooth data transmission module. The force plate had local intelligence in order to choose the information to send to the LPU, using proprietary software that cannot be modified remotely. The force plate was battery powered, with measurement time set to 10 seconds. In order to aid energy conservation and to avoid any perturbation of the measurement, data transmission is performed at the end of the recording period. The force plate remains on standby, and is activated by the approach of the person using a presence sensor.

Upon activation, the force plate starts measurement and memorization. Once the force values pass a threshold, indicating that someone is on the force plate, the timer starts for the static measurement. At the end of the predetermined duration for static measurement, an indicator is activated to signify that the person can step down from the force plate. Data recording stops at the end of the measurement period and the COP signals are calculated, before being transmitted via Bluetooth to the local processing unit. After data transmission, the force plate disconnects and returns to standby.

In contrast to the force plate, the camera will perform image processing, in order to ensure that no images of the person will be transmitted, thus protecting the privacy of the person being observed. Instead of images, parameters related to gait quality will be sent to the LPU.

In the present application, the LPU is a mobile phone (6600, Nokia, Finland), with programs written in Java. The functions contained in the LPU include signal processing and extraction of relevant parameters from the force plate signals using algorithms based on clinical data [50,69], reception of the gait analysis parameters [64], and a decision making capability. The LPU also communicates with both the sensors, and the rest of the surveillance network. The LPU can transmit the decision outcome by SMS or by Internet using GSM technology.

The LPU has three processes that survey the arrival of data. The first surveys Bluetooth to detect the arrival of the data from the force plate and integrates the data processing functions. This process waits for a request for a Bluetooth connection, receives the data for storage in memory, before closing the connection. The signature is then calculated from these data and is compared with previous data to determine if there has been any change. Depending on the results of the test, an SMS can be sent to a neighbor, a friend, a family member, or a possible sociomedical network. Thereafter, a connection can be established with a remote server in order to transmit the latest results that are stored in the database.

The second process surveys Bluetooth to detect the arrival of the data from the camera. This process functions as for the force plate, but without the requirement for data processing.

The third process surveys the telephone connection in order to respond to any modification of the detection parameters by the remote system, with priority given to the first process. When the server receives a demand for an update, a connection is established with the mobile phone via GSM and the parameters are updated. The new parameters are initially stored in a FIFO (first input first output), before the telephone is disconnected. The new parameters are stored as soon as it is possible to access the common memory, before the process returns to its initial state, waiting for a new call.

All of the actors in the network are linked to a dedicated server. The four different types of actors in the network use a communication type that varies according to their function. The local support network (family, friends, and neighbors) and emergency services receive information via phone or SMS, whereas the medical support network (doctors, nurses, etc.) receives more detailed information by Internet. The telecommunications service communicates with the LPU to verify functioning and update programs and decision parameters.

The system created is nonintrusive, low cost, and easily adaptable, thanks to the JAVA technology, and can be controlled remotely. The local installation part of the system has been tested successfully in a laboratory setting for both Internet and SMS communication. Telecommunications are now being evaluated, in particular the update facility, after which a field trial of remote assessment of balance in elderly subjects will begin. Such data is needed to adapt the treatment algorithms, and to evaluate the efficacy of the system.

\section{CONCLUSION}

The aim of the project was to develop a methodology and a technology that would enable the detection of an evolution towards a risk of falling in community-living elderly. To this end, it has been demonstrated that it is possible to obtain parameters characterizing static and dynamic equilibrium from a force plate, with no constraint on the posture of the person measured. In addition, it has been shown that 
a simple video camera is capable of following the movements of a person without requiring any markers or reflectors. The model, thus obtained, enables the calculation of kinematic and spatiotemporal variables already identified as pertinent in an analysis of the risk of falling.

Despite the advances made, there are a number of challenges still remaining. Firstly, it will be necessary to develop a methodology to exploit the information provided by the two sensors synergistically in order to make a decision related to the behavior of the individual studied. This decision will need to be based on a self-learning process, with each subject as its own reference. Data fusion requires the simultaneous use of several different data sources in order to obtain better-quality information. In order to obtain the best possible results, it will be necessary to have large quantities of clinical data from both systems. To this end, the second aspect of the followup to the PARAChute project is to perform a clinical study to establish the medical validity of the approach adopted. An initial validation is already underway in a controlled environment, while a longitudinal study is scheduled for 2007 and 2008. This latter study will run for two years using diverse sites in order to offer the possibility of analyzing different study populations with different levels of balance impairment.

\section{ACKNOWLEDGMENTS}

This study was undertaken as part of the PARAChute research project (Personnes Âgées et Risque de Chute), which was supported in part by The French Ministry of Research (Grant 03-B-254), The European Social Fund (Grant 3/1/3/4/07/3/3/011), The European Regional Development Fund (Grant 2003-2-50-0014), The Champagne-Ardenne Regional Council (Grant E200308251), and INRIA (Grant 804F04620016000081).

\section{REFERENCES}

[1] Comité Français d’Education pour la Santé, "Les clés du "bien vieillir": prévention des chutes chez les seniors," Caisse Nationale de l'Assurance Maladie des Travailleurs Salariés, p. 20, 2001.

[2] S. G. Brauer, Y. R. Burns, and P. Galley, "A prospective study of laboratory and clinical measures of postural stability to predict community-dwelling fallers," The Journals of Gerontology Series A: Biological Sciences and Medical Sciences, vol. 55, no. 8, pp. M469-M476, 2000.

[3] B. E. Maki, P. J. Holliday, and A. K. Topper, "A prospective study of postural balance and risk of falling in an ambulatory and independent elderly population," Journal of Gerontology, vol. 49, no. 2, pp. M72-M84, 1994.

[4] M. Raîche, R. Hébert, F. Prince, and H. Corriveau, "Screening older adults at risk of falling with the Tinetti balance scale," The Lancet, vol. 356, no. 9234, pp. 1001-1002, 2000.

[5] A. M. Tromp, S. M. F. Pluijm, J. H. Smit, D. J. H. Deeg, L. M. Bouter, and P. Lips, "Fall-risk screening test: a prospective study on predictors for falls in community-dwelling elderly," Journal of Clinical Epidemiology, vol. 54, no. 8, pp. 837-844, 2001.
[6] L. Z. Rubenstein and K. R. Josephson, "The epidemiology of falls and syncope," Clinics in Geriatric Medicine, vol. 18, no. 2, pp. 141-158, 2002.

[7] M. C. Nevitt, S. R. Cummings, S. Kidd, and D. Black, "Risk factors for recurrent nonsyncopal falls. A prospective study," The Journal of the American Medical Association, vol. 261, no. 18, pp. 2663-2668, 1989.

[8] M. E. Tinetti, M. Speechley, and S. F. Ginter, "Risk factors for falls among elderly persons living in the community," New England Journal of Medicine, vol. 319, no. 26, pp. 1701-1707, 1988.

[9] B. J. Vellas, S. J. Wayne, L. Romero, R. N. Baumgartner, L. Z. Rubenstein, and P. J. Garry, "One-leg balance is an important predictor of injurious falls in older persons," Journal of the American Geriatrics Society, vol. 45, no. 6, pp. 735-738, 1997.

[10] B. H. Wood, J. A. Bilclough, A. Bowron, and R. W. Walker, "Incidence and prediction of falls in Parkinson's disease: a prospective multidisciplinary study," Journal of Neurology Neurosurgery and Psychiatry, vol. 72, no. 6, pp. 721-725, 2002.

[11] J. H. J. Allum, B. R. Bloem, M. G. Carpenter, and F. Honegger, "Differential diagnosis of proprioceptive and vestibular deficits using dynamic support-surface posturography," Gait \& Posture, vol. 14, no. 3, pp. 217-226, 2001.

[12] J. Gill, J. H. J. Allum, M. G. Carpenter, et al., "Trunk sway measures of postural stability during clinical balance tests: effects of age," The Journals of Gerontology Series A: Biological Sciences and Medical Sciences, vol. 56, no. 7, pp. M438-M447, 2001.

[13] S. R. Lord and H. B. Menz, "Visual contributions to postural stability in older adults," Gerontology, vol. 46, no. 6, pp. 306310,2000 .

[14] S. Mathias, U. S. L. Nayak, and B. Isaacs, "Balance in elderly patients: the "get-up and go" test," Archives of Physical Medicine and Rehabilitation, vol. 67, no. 6, pp. 387-389, 1986.

[15] A. Shumway-Cook, M. Baldwin, N. L. Polissar, and W. Gruber, "Predicting the probability for falls in community-dwelling older adults," Physical Therapy, vol. 77, no. 8, pp. 812-819, 1997.

[16] S. R. Lord, R. D. Clark, and I. W. Webster, "Postural stability and associated physiological factors in a population of aged persons," Journal of Gerontology, vol. 46, no. 3, pp. M69-76, 1991.

[17] P. B. Thapa, P. Gideon, K. G. Brockman, R. L. Fought, and W. A. Ray, "Clinical and biomechanical measures of balance as fall predictors in ambulatory nursing home residents," The Journals of Gerontology Series A: Biological Sciences and Medical Sciences, vol. 51, no. 5, pp. M239-M246, 1996.

[18] S. A. Niyogi and E. H. Adelson, "Analyzing and recognizing walking figures in XYT," in Proceedings of the IEEE Computer Society Conference on Computer Vision and Pattern Recognition (CVPR '94), pp. 469-474, Seattle, Wash, USA, June 1994.

[19] P. S. Huang, C. J. Harris, and M. S. Nixon, "Comparing different template features for recognizing people by their gait," in Proceedings of the 9th British Machine Vision Conference (BMVA '98), vol. 2, pp. 639-648, Southampton, UK, September 1998.

[20] A. Kale, A. N. Rajagopalan, N. Cuntoor, and V. Kruger, "Gaitbased recognition of humans using continuous HMMs," in Proceedings of the 5th IEEE International Conference on Automatic Face and Gesture Recognition, pp. 321-326, Washington, DC, USA, May 2002.

[21] D. Meyer, "Human gait classification based on hidden Markov model," in 3D Image Analysis and Synthesis, pp. 139-146, Sankt Augustin, Germany, November 1997. 
[22] C. Ben Abdelkader, R. Cutler, and L. S. Davis, "View-invariant estimation of height and stride for gait recognition," in Proceedings of the International European Conference on Computer Vision Workshop Copenhagen on Biometric Authentication, pp. 155-167, Copenhagen, Denmark, June 2002.

[23] N. V. Boulgouris, K. N. Plataniotis, and D. Hatzinakos, "Gait recognition using linear time normalization," Pattern Recognition, vol. 39, no. 5, pp. 969-979, 2006.

[24] Y. Liu, R. Collins, and Y. Tsin, "Gait sequence analysis using Frieze patterns," Tech. Rep. CMU-RI-TR-01-38, Robotics Institute, Carnegie Mellon University, Pittsburgh, Pa, USA, December 2001.

[25] J. Close, M. Ellis, R. Hooper, E. Glucksman, S. Jackson, and C. Swift, "Prevention of falls in the elderly trial (PROFET): a randomised controlled trial," The Lancet, vol. 353, no. 9147, pp. 93-97, 1999.

[26] R. G. Cumming, M. Thomas, G. Szonyi, et al., "Home visits by an occupational therapist for assessment and modification of environmental hazards: a randomized trial of falls prevention," Journal of the American Geriatrics Society, vol. 47, no. 12, pp. 1397-1402, 1999.

[27] D. M. Buchner, M. E. Cress, B. J. de Lateur, et al., "The effect of strength and endurance training on gait, balance, fall risk, and health services use in community-living older adults," The Journals of Gerontology Series A: Biological Sciences and Medical Sciences, vol. 52, no. 4, pp. M218-M224, 1997.

[28] S. L. Wolf, H. X. Barnhart, N. G. Kutner, E. McNeely, C. Coogler, and $\mathrm{T}$. $\mathrm{Xu}$, "Reducing frailty and falls in older persons: an investigation of Tai Chi and computerized balance training. Atlanta FICSIT Group. Frailty and Injuries: Cooperative Studies of Intervention Techniques," Journal of the American Geriatrics Society, vol. 44, no. 5, pp. 489-497, 1996.

[29] A. J. Campbell, M. C. Robertson, M. M. Gardner, R. N. Norton, and D. M. Buchner, "Falls prevention over 2 years: a randomized controlled trial in women 80 years and older," Age and Ageing, vol. 28, no. 6, pp. 513-518, 1999.

[30] M. C. Robertson, N. Devlin, P. Scuffham, M. M. Gardner, D. M. Buchner, and A. J. Campbell, "Economic evaluation of a community based exercise programme to prevent falls," Journal of Epidemiology and Community Health, vol. 55, no. 8, pp. 600-606, 2001.

[31] A. H. Myers, Y. Young, and J. A. Langlois, "Prevention of falls in the elderly," Bone, vol. 18, no. 1, supplement 1, pp. $87 \mathrm{~S}-$ 101S, 1996.

[32] J. A. Rizzo, R. Friedkin, C. S. Williams, J. Nabors, D. Acampora, and M. E. Tinetti, "Health care utilization and costs in a Medicare population by fall status," Medical Care, vol. 36, no. 8, pp. 1174-1188, 1998.

[33] S. L. Whitney, G. F. Marchetti, and A. I. Schade, "The relationship between falls history and computerized dynamic posturography in persons with balance and vestibular disorders," Archives of Physical Medicine and Rehabilitation, vol. 87, no. 3, pp. 402-407, 2006.

[34] J. E. Zachazewski, P. O. Riley, and D. E. Krebs, "Biomechanical analysis of body mass transfer during stair ascent and descent of healthy subjects," Journal of Rehabilitation Research and Development, vol. 30, no. 4, pp. 412-422, 1993.

[35] A. Stacoff, C. Diezi, G. Luder, E. Stüssi, and I. A. Kramersde Quervain, "Ground reaction forces on stairs: effects of stair inclination and age," Gait \& Posture, vol. 21, no. 1, pp. 24-38, 2005.

[36] R. K. Begg and W. A. Sparrow, "Gait characteristics of young and older individuals negotiating a raised surface: implications for the prevention of falls," The Journals of Gerontology
Series A: Biological Sciences and Medical Sciences, vol. 55, no. 3, pp. M147-M154, 2000.

[37] K. A. Hamel, N. Okita, J. S. Higginson, and P. R. Cavanagh, "Foot clearance during stair descent: effects of age and illumination," Gait \& Posture, vol. 21, no. 2, pp. 135-140, 2005.

[38] B. J. McFadyen and D. A. Winter, "An integrated biomechanical analysis of normal stair ascent and descent," Journal of Biomechanics, vol. 21, no. 9, pp. 733-744, 1988.

[39] Y. Laufer, "Effect of age on characteristics of forward and backward gait at preferred and accelerated walking speed," The Journals of Gerontology Series A: Biological Sciences and Medical Sciences, vol. 60, no. 5, pp. 627-632, 2005.

[40] T. E. Prieto, J. B. Myklebust, R. G. Hoffmann, E. G. Lovett, and B. M. Myklebust, "Measures of postural steadiness: differences between healthy young and elderly adults," IEEE Transactions on Biomedical Engineering, vol. 43, no. 9, pp. 956-966, 1996.

[41] J. J. Collins and C. J. De Luca, "Open-loop and closedloop control of posture: a random-walk analysis of centerof-pressure trajectories," Experimental Brain Research, vol. 95, no. 2, pp. 308-318, 1993.

[42] D. Delignières, T. Deschamps, A. Legros, and N. Caillou, "A methodological note on nonlinear time series analysis: is the open- and closed-loop model of Collins and De Luca (1993) a statistical artifact?" Journal of Motor Behavior, vol. 35, no. 1, pp. 86-96, 2003.

[43] A. L. Goldberger, L. A. N. Amaral, J. M. Hausdorff, P. Ch. Ivanov, C.-K. Peng, and H. E. Stanley, "Fractal dynamics in physiology: aterations with disease and aging," Proceedings of the National Academy of Sciences of the United States of America, vol. 99, supplement 1, pp. 2466-2472, 2002.

[44] C. K. Peng, S. Havlin, H. E. Stanley, and A. L. Goldberger, "Quantification of scaling exponents and crossover phenomena in nonstationary heartbeat time series," Chaos, vol. 5, no. 1, pp. 82-87, 1995.

[45] A. Eke, P. Herman, L. Kocsis, and L. R. Kozak, "Fractal characterization of complexity in temporal physiological signals," Physiological Measurement, vol. 23, no. 1, pp. R1-R38, 2002.

[46] P. E. Shrout and J. L. Fleiss, "Intraclass correlations: uses in assessing rater reliability," Psychological Bulletin, vol. 86, no. 2, pp. 420-428, 1979.

[47] J. A. Norris, A. P. Marsh, I. J. Smith, R. I. Kohut, and M. E. Miller, "Ability of static and statistical mechanics posturographic measures to distinguish between age and fall risk," Journal of Biomechanics, vol. 38, no. 6, pp. 1263-1272, 2005.

[48] S. Nadeau, B. J. McFadyen, and F. Malouin, "Frontal and sagittal plane analyses of the stair climbing task in healthy adults aged over 40 years: what are the challenges compared to level walking?” Clinical Biomechanics, vol. 18, no. 10, pp. 950-959, 2003.

[49] T. Gélat and Y. Brenière, "Adaptation of the gait initiation process for stepping on to a new level using a single step," Experimental Brain Research, vol. 133, no. 4, pp. 538-546, 2000.

[50] V. Michel, H. Amoud, D. J. Hewson, J.-Y. Hogrel, and J. Duchêne, "The effect of age on dynamic postural equilibrium when stepping up," in Proceedings of the 17th Conference of the International Society for Postural and Gait Research (ISPGR '05), Marseille, France, May-June 2005.

[51] V. Michel, D. J. Hewson, J.-Y. Hogrel, and J. Duchêne, "Analysis of postural dynamic equilibrium in elderly subjects descending a stair backwards," in Proceedings of the 3rd European Medical and Biological Engineering Conference (EMBEC): Advancement of Medicine and Health Care through Technology, Prague, Czech Republic, November 2005. 
[52] J. L. Fleiss, The Design and Analysis of Clinical Experiments, John Wiley \& Sons, New York, NY, USA, 1986.

[53] D. Lafond, H. Corriveau, R. Hébert, and F. Prince, "Intrasession reliability of center of pressure measures of postural steadiness in healthy elderly people," Archives of Physical Medicine and Rehabilitation, vol. 85, no. 6, pp. 896-901, 2004.

[54] R. A. Du Pasquier, Y. Blanc, M. Sinnreich, T. Landis, P. Burkhard, and F. J. G. Vingerhoets, "The effect of aging on postural stability: a cross sectional and longitudinal study," Neurophysiologie Clinique/Clinical Neurophysiology, vol. 33, no. 5, pp. 213-218, 2003.

[55] L. Chiari, A. Cappello, D. Lenzi, and U. Della Croce, "An improved technique for the extraction of stochastic parameters from stabilograms," Gait \& Posture, vol. 12, no. 3, pp. 225-234, 2000.

[56] H. Corriveau, R. Hébert, F. Prince, and M. Raîche, "Postural control in the elderly: an analysis of test-retest and interrater reliability of the COP-COM variable," Archives of Physical Medicine and Rehabilitation, vol. 82, no. 1, pp. 80-85, 2001.

[57] B. Schölkopf, J. C. Platt, J. Shawe-Taylor, A. J. Smola, and R. C. Williamson, "Estimating the support of a high-dimensional distribution," Neural Computation, vol. 13, no. 7, pp. 14431471, 2001.

[58] B. Scholkopf, R. C. Williamson, A. J. Smola, J. Shawe-Taylor, and J. C. Platt, "Support vector method for novelty detection," in Advances in Neural Information Processing Systems, vol. 12, pp. 582-588, MIT Press, Cambridege, Mass, USA, 2000.

[59] D. M. J. Tax, One-class classification, Ph.D. thesis, Delft University of Technology, Delft, The Netherlands, 2001.

[60] D. M. J. Tax and R. P. W. Duin, "Support vector domain description," Pattern Recognition Letters, vol. 20, no. 11-13, pp. 1191-1199, 1999.

[61] D. M. J. Tax and R. P. W. Duin, "Support vector data description," Machine Learning, vol. 54, no. 1, pp. 45-66, 2004.

[62] V. N. Vapnik, Statistical Learning Theory, John Wiley \& Sons, New York, NY, USA, 1998.

[63] V. Michel-Pelligrino, H. Amoud, D. J. Hewson, and J. Duchêne, "Identification of a degradation in postural equilibrium invoked by different vibration frequencies on the tibialis anterior tendon," in Proceedings of the 28th Annual International Conference of the IEEE Engineering in Medicine and Biology Society (EMBS '06), pp. 4047-4050, New York, NY, USA, August 2006.

[64] J. Saboune and F. Charpillet, "Using interval particle filtering for marker less 3D human motion capture," in Proceedings of the 17th IEEE International Conference on Tools with Artificial Intelligence (ICTAI '05), pp. 621-627, Hong Kong, November 2005.

[65] J. Deutscher, A. Blake, and I. Reid, "Articulated body motion capture by annealed particle filtering," in Proceedings of the IEEE Computer Society Conference on Computer Vision and Pattern Recognition (CVPR '00), vol. 2, pp. 126-133, Hilton Head Island, SC, USA, June 2000.

[66] J. M. Hausdorff, Y. Ashkenazy, C.-K. Peng, P. Ch. Ivanov, H. E. Stanley, and A. L. Goldberger, "When human walking becomes random walking: fractal analysis and modeling of gait rhythm fluctuations," Physica A: Statistical Mechanics and Its Applications, vol. 302, no. 1-4, pp. 138-147, 2001.

[67] T. Herman, N. Giladi, T. Gurevich, and J. M. Hausdorff, "Gait instability and fractal dynamics of older adults with a "cau- tious" gait: why do certain older adults walk fearfully?” Gait \& Posture, vol. 21, no. 2, pp. 178-185, 2005.

[68] M. Doussot, D. J. Hewson, and J. Duchêne, "A remote monitoring system for assessing balance quality," in Proceedings of the 3rd European Medical and Biological Engineering Conference (EMBEC): Advancement of Medicine and Health Care through Technology, Prague, Czech Republic, November 2005.

[69] H. Amoud, M. Abadi, V. Michel, D. J. Hewson, M. Doussot, and J. Duchêne, "Evaluation de l'équilibre postural suite à la réalisation d'un pas selon trois méthodes d'estimation de l'exposant de Hurst," in 20e colloque GRETSI sur le traitement du signal et des images (GRETSI '05), Louvain-la-Neuve, Belguim, September 2005.

David J. Hewson worked as a Research Physiologist for the Royal New Zealand Air Force between 1994 and 2000, and received his Ph.D. degree from the University of Auckland in 2000. He is now an Associate Professor at the University of Technology of Troyes. His research interests are ergonomic and clinical applications of surface electromyography, and the development of new methodologies for fall prevention in the elderly. He is the Coordinator and Chief Investigator of the PARAChute and PréDICA projects on the prevention of falls in the elderly.

Jacques Duchêne received the Engineer degree in electronics from the Ecole Supérieure d'Electricité (France) in 1973, and the Doctorat d'État in sciences in 1983. He joined the University of Technology of Troyes in 1994, where he is currently in charge of the Charles Delaunay Institute of Research. His main research interests are signal processing, pattern recognition, and classification. He now focuses on signal

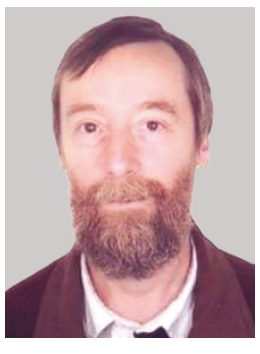
segmentation as well as signal decomposition. His main application fields in biomedical engineering are ergonomics (comfort in cars), biomedical monitoring (quality of balance in the elderly), and EMG characterization and modelling (frequency parameters, conduction velocity distribution).

François Charpillet is currently a Director of Research at the National Institute of Research in Automatics and Computer science (INRIA). He received a Ph.D. degree in computer science (on speech recognition) from the University Henri Poincaré, Nancy 1, in 1985, and an HDR from the same university in 1998. He is also an Alumnus of Ecole Normale Supérieure d'Electricité et de Mécanique. He has been the Leader of the

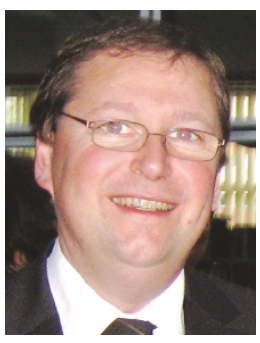
Project/Team MAIA in Nancy since 1998 that is part of both the LORIA Laboratory and INRIA. This group of around 25 members is interested in artificial intelligence focusing on sequential and/or distributed decision under uncertainty. He has taken part in various national programs in the field of healthcare, including patient follow-up after kidney transplantation, fall prevention in the elderly, segmentation of electrocardiogram signals, telemonitoring of kidney disease patients, and early detection of cutaneous infection of patients undergoing peritoneal dialysis. 
Jamal Saboune received an Engineering Diploma in electrical and electronic engineering from the Faculty of Engineering of the Lebanese University in 2002. In 2003, he obtained a Research Masters degree in information and system technologies from the Universite de Technologie de Compiègne (UTC), France. He is currently a Ph.D. student at the Universite de Technologie de Troyes (UTT), France. He is pursuing his research activities as a Member of the MAIA Team of INRIA in Nancy, France.

Valérie Michel-Pellegrino received her Ph.D. degree in biology: biomechnics and movement physiology in 2003 from the University of Paris XI (France). She worked with Manh-Cuong DO (director of research for both M.S. and Ph.D. degrees) at Laboratory of Movement Physiology, Paris XI, France, from 1999 to 2003. Since 2004, she has been associated with the "Laboratoire de Modélisation et Sûreté

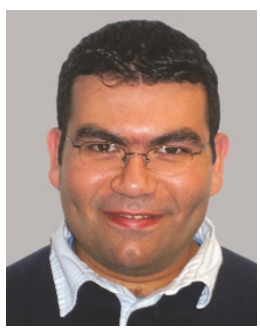
des Systèmes" of the Institut Charles Delaunay Troyes, France and she works with Jacques Duchêne and David Hewson. Her research interest focuses on the neurophysiology and the biomechanics of the control of movement and posture. More precisely, she analyzes the reorganization of the motor process developed to control balance during a locomotor task in elderly as well as pathological people.

Hassan Amoud received an Engineering degree in computer science from the Lebanese University, Lebanon, in 2002, before obtaining his M.S. degree in 2003 from the University of Technology of Compiègne, France. He completed his Ph.D. degree in signal processing and biomedical engineering from the University of Technology of Troyes (UTT), France, in 2006, on the detection of an evolution in the risk of falls

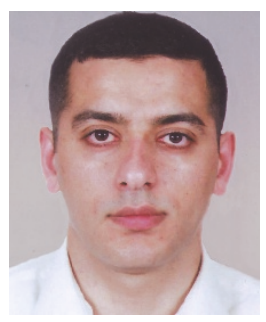
in the elderly. He is currently a Research Fellow at the UTT. His research work is focused on signal processing, biomedical engineering, data analysis, and postural control. He has worked on French research projects such as PARAChute (risk of falls in the elderly).

Michel Doussot received an M.S. degree in electronic and automatic engineering from the University of Nancy (France) in 1989, and a Ph.D. degree in automatic and signal processing from the University of Reims (France) in 1994. He joined the University of Technology of Troyes in 1998. His main competences and interests are in signal processing and real-time embedded systems. He currently works on sensor networks and

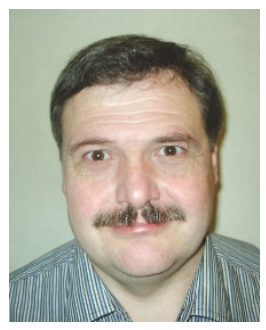
on the integration of sensors and wireless device in ASIC. He also works on the optimization of wireless communication protocols for energy economy.
Jean Paysant is currently an Associate Professor at the Université Henri Poincaré Medical School, Nancy, France, and a Hospital Practitioner specializing in rehabilitation and physical medicine. He is accredited by the European Board of Physical Medicine and Rehabilitation, and has also completed an M.S. degree in biomechanics and movement physiology from the University of Paris XI, and a Ph.D. degree in health

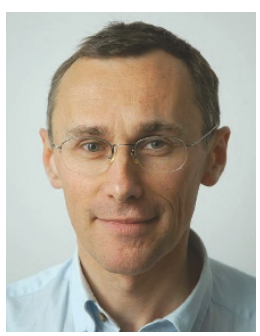
science from the University of Dijon. His research interests include gait analysis (kinematics, kinetics, and kinesiology) of both normal and pathological subjects, including lower limb amputation, cerebral palsy, and polytraumatism; ambulatory monitoring for motor performance (accelerometry, electrogoniometry), and amputation and prosthetics (technology, compensatory mechanisms, capacity, and activity).

Anne Boyer is currently an Associate Professor in computer science at LORIAINRIA, Nancy, France. Her research interests are related to the statistical analysis of daily practices in order to model the comportment of the user. Her main domains of application are telemedicine information research on the Internet.

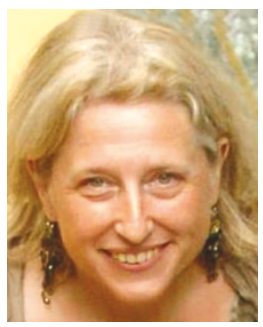

Jean-Yves Hogrel received the B.S. degree, the M.S. degree (1990), and the Ph.D. degree (1994) in biomedical engineering from the University of Technology of Compiègne, France. In 1995, he joined the Neuromuscular Functional Exploration Center at the Institute of Myology (Pitié-Salpêtrière Hospital, Paris), where he is currently the Head of the Neuromuscular Physiology and Evaluation Lab. His research interests mainly focus

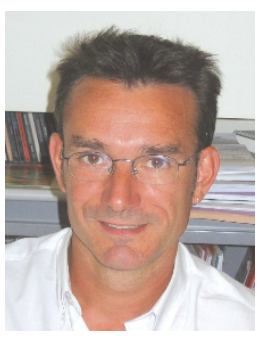
on the clinical use of surface electromyography (detection, signal processing, and data analysis) and more generally in the quantified evaluation of patients suffering from neuromuscular disorders (diagnostic, therapeutic trials, and assessment in clinical routine of the neuromuscular function). 